\title{
Evaluación psicométrica de un instrumento para la medición de variables asociadas al consumo electrónico en el contexto venezolano*
}

\author{
Measurement Variables Associated with Electronic Consumption \\ in Venezuela Context: Psychometric Examination
}

Recibido: mayo 22 de 2009 ～Revisado: abril 12 de 2010 | Aceptado: octubre 4 de 2010

\author{
SofíA PeINADO** \\ AlejandRa SALAS \\ MARÍA LORENA CAMPOS U. \\ Universidad Simón Bolívar, Caracas, Venezuela
}

Para citar este artículo. Peinado, S., Salas, A., \& Campos, M. L. (2011). Evaluación psicométrica de un instrumento para la medición de variables asociadas al consumo electrónico en el contexto venezolano. Universitas Psychologica, 10(1), 211229.

* Artículo de investigación.

** Dpto. Ciencia y Tecnología del Comportamiento. Edificio EGE, Piso 1. Sartenejas, Baruta, Estado Miranda. Apartado postal No.89.000 Caracas (Venezuela). Teléfonos: (0058212) 906-3779; 906-3819; 906-3821, Celular (0058412) 3143725. E-mails: speinado@usb.ve; alesalas@usb.ve; camposl@usb. ve

\section{RES UMEN}

Esta investigación se propuso analizar la validez y confiabilidad de un instrumento para medir variables asociadas al consumo electrónico: Percepción de Utilidad (PU), Autoeficacia Computacional (AC), Intención de Consumo (IC) y Consumo Electrónico (CE), a partir de la adaptación de las escalas utilizadas por Cruz García (2007a, 2007b) y sugerir adaptaciones del mismo para el entorno venezolano. 214 estudiantes de posgrado constituyeron la muestra. Esta investigación condujo a un nuevo instrumento: Inventario de Variables Asociadas al Consumo Electrónico (IVACE), cuyo análisis psicométrico demostró que las cuatro escalas tienen altos niveles de confiabilidad ( $\propto \geq 80)$. El análisis factorial evidenció que las escalas de IC y CE tienen una dimensión, y las escalas de PUy AC revelaron dos dimensiones. Palabras claves autores

Consumo electrónico, percepción de utilidad, intención de compra, autoeficacia computacional.

Palabras clave descriptores

Psicometría, comercio electrónico, Venezuela, consumo electrónico.

\section{A B S T R A C T}

The purpose of this research was statistical test (validity and reliability) of Electronic Consumption Questionnaire, this included following subscales: Perceived Usefulness, Computational Self-efficacy, Consumption Intention, Electronic Consumption, were adapted from Garcia's model (2007a $\&$ 2007b), and make suggest to adapt it a Venezuela context. 214 students answered the questionnaire. The new instrument was named IVACE (Variables associated with Electronic Consumption Inventory), psychometric analysis resulted high reliability level $(\propto \geq 0.80)$. Factors analysis showed Consumption Intention and Electronic Consumption scales had a single dimension, the others scales, Perceived Usefulness and Computational Self-efficacy have two dimensions each.

Key words authors

Electronic consumption, consumption intention, perceived usefulness, computational self-efficacy.

Key words plus

Psychometrics, electronic commerce, electronic consumption, Venezuela. 


\section{Introducción}

La incorporación de las Tecnologías de la Información y la Comunicación (TIC) en la economía, ha generado una nueva forma de consumo, puesto que han surgido nuevos bienes de así como nuevas formas de obtener lo que se desea consumir. A este último fenómeno responde el desarrollo del comercio electrónico que, en América Latina gracias al aumento del acceso por banda ancha, ha crecido en forma sostenida (Empresa Exterior [EFE], 2008a). Según el director de la oficina de Google para América Latina, la cantidad de latinoamericanos que usan la web se duplicó en los últimos cuatro años (EFE, 2008a); este aumento llevo a un crecimiento de las transacciones comerciales en línea, cuyo valor superó los $\$ 1.800$ millones en el 2007. Ahora bien, dichas transacciones comerciales son solo a una parte de los bienes económicos que pueden obtenerse por esa vía, ya que en línea se pueden recibir numerosos servicios de manera gratuita. En tal sentido Alonso señala el aumento de las personas que deciden comprar a través de Internet, si bien no siempre la transacción comercial se concreta en línea. Por esta razón, este trabajo se centra en el estudio del consumo electrónico, definido como la acción que procura satisfacer una necesidad u obtener un beneficio a través del uso de la web, independientemente de que se deba pagar por ello.

La noción de consumo electrónico fue escogida por ser una acepción más amplia que la contenida en el término comercio electrónico, que Iriarte (s. f.) define como "la adquisición de un bien o un servicio, que utiliza los medios electrónicos tanto para alguna parte o todo el proceso del acto de comercio" ( $(2)$. Analizar el consumo en lugar del comercio permite incluir numerosas transacciones que las personas realizan de manera gratuita en sitios web y que son financiados por medio de la publicidad; en cuyo caso, quien paga el servicio publicitario subvenciona, indirectamente, el consumo realizado por quienes utilizan los servicios gratuitos ofrecidos en el sitio. El tipo de consumo apenas descrito podría explicar las diferencias de hasta 43,9 millones de personas, que aun habiendo sido clasificadas como usuarios on-line en América Latina, no son considerados compradores on-line (Master-Net.net, 2007).

Según los informes elaborados por Pyramid Research y Americaeconomía Intelligence, Venezuela lidera el crecimiento latinoamericano de usuarios de la web, pasando de 316 mil a 1,08 millones de usuarios registrados entre 2003 y 2007 y llevando al alza de $224 \%$ de su comercio electrónico en el 2007, muy por encima del $121 \%$ promedio de la región (EFE, 2008a, 2008b). Aún, ante este crecimiento significativo de usuarios y del comercio electrónico, en Venezuela son escasas las investigaciones que permitan comprender el fenómeno del consumo electrónico.

Las estadísticas sobre el comercio electrónico en Venezuela indicarían que, ya en el 2004, cerca del 50\% de las empresas contaban con infraestructura para hacer negocios en línea, y que estos superaban, para entonces, 800 millones de dólares, los internautas locales realizaron compras al detal por Internet de 42 millones de dólares en el año 2003, y el crecimiento del comercio electrónico era liderado por el sector bancario que ofrecía productos a costos competitivos (Gómez, 2004).

El caso venezolano merece hoy más atención que antes, puesto que existen condiciones económicas particulares que pudieran haber contribuido al crecimiento extraordinario del comercio electrónico: el control cambiario que genera una revaloración del cambio de la moneda local frente al dólar, el alto índice inflacionario, las tasas reales negativas de las tarjetas de crédito y la disminución de la producción manufacturera local. ¿Podrían estas circunstancias económicas afectar la interacción de las variables que determinan el consumo electrónico? Para responder a esta pregunta, convendría comparar estudios realizados en diferentes países de la región; sin embargo, para que dichas comparaciones sean válidas, es conveniente adaptar los instrumentos de medición al contexto de su aplicación para así asegurar su validez y confiabilidad.

A través del presente estudio, se pretende, entonces, contribuir a la comprensión del comportamiento del consumidor venezolano en el entorno 
electrónico, planteando los siguientes objetivos: (a) analizar la validez y confiabilidad de un instrumento para medir variables asociadas al consumo electrónico, a partir de la adaptación de las escalas utilizadas por García (2007a) para plantear su Modelo del Consumo Electrónico (MCE) y (b) sugerir modificaciones o adaptaciones del mismo para mejorar su eficacia en el entorno venezolano.

\section{Marco referencial}

El Modelo de Aceptación de la Tecnología (TAM, por sus siglas en inglés), propuesto por Fred Davis en su disertación doctoral de presentada en 1986 en el Sloan School of Management, es una herramienta que surgió para estimar y predecir la aceptación del usuario de las tecnologías informáticas (TI) emergentes que habían ganado popularidad (Davis, 1989). Este modelo fue desarrollado bajo un contrato IBM Canadá Ltd., a mediados de 1980, y fue usado para evaluar el mercado potencial de una variedad de nuevas aplicaciones basadas en PC en el ámbito de la multimedia, procesamiento de imágenes y para orientar las inversiones en actividades de desarrollo (Davis \& Venkatesch, 1995).

El TAM es una adaptación de la Teoría de Acción Razonada (TAR) (Ajzen \& Fishbein, 1980); que toma en consideración solamente dos de las creencias conductuales específicas de la TAR: la Utilidad Percibida (UP) y la Percepción de Facilidad de Uso (PFU) que determinan la intención conductual del individuo para usar TI:

- La PFU "es definida como el grado en el cual una persona cree que el uso de un sistema en particular estará libre de esfuerzo" (Davis, 1989, p. 320).

- La UP es "el grado en que una persona cree que el uso de un sistema puede mejorar su desempeño" (Davis, 1989, p. 320).

El instrumento original para medir estas creencias fue desarrollado y validado por Davis en 1994; ha sido replicado exitosamente y usado extensivamente por investigadores en el área de aceptación de los usuarios. Registrando en la mayoría de los ca- sos, una confiabilidad > 0.9 (alpha de Cronbach) para la escala de UP y la escala de PFU, estas han mostrado también un alto grado de convergencia y validez discriminante (Davis \& Venkatesch, 1995).

Investigaciones empíricas han evaluado el poder explicativo del TAM y su validez de medición en diferentes contextos en base a constructos, a tipo de sistemas de información (SI), correo electrónico, procesadores de palabras, programas gráficos, computadores personales, y otros programas. Estudios recientes han evaluado el uso del Modelo para analizar la conducta de los usuarios en Internet, específicamente WWW (Lederer, Maupin, Sena \& Zhuang, 2000; Teo, Lim \& Lai, 1999).

El constructo actitud fue descartado del TAM, porque la experiencia empírica demostró que no mediaba entre el efecto de la UP y la intención conductual (Venkatesh, 1999 citado en Lee, Park \& Ahn, 2000). Así mismo, estos autores proponen una adaptación del modelo TAM (Figura 1).

\section{Figura 1}

Adaptación de TAM

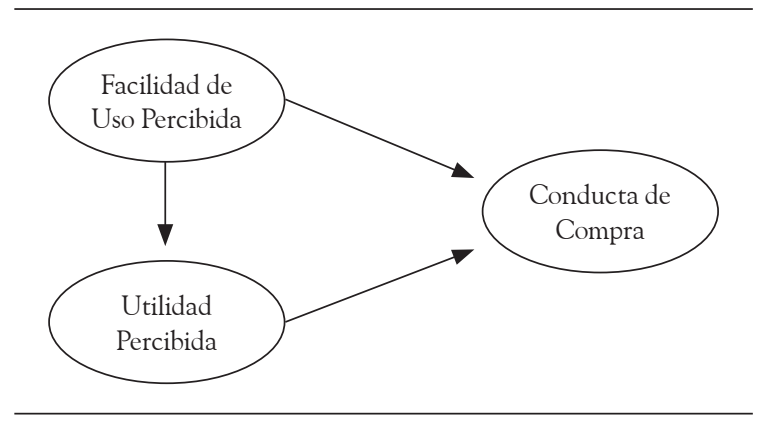

Fuente: propuesto por Lee, Park \& Ahn, 2000.

Otros autores incluyen variables adicionales a las del TAM, tales como: el riesgo percibido con el producto/servicio, el riesgo percibido por un consumidor en una situación particular de compra Cox y Rich (1964); otros mencionan los sentimientos (Chaudri, 1998 citado en Lee, Park \& Ahn, 2000) y las expectativas (Sweeney, Soutar \& Johnson, 1999 citados en Lee, Park \& Ahn, 2000) como mediadores del riesgo percibido. La inclusión de estas variables han generado adaptaciones del TAM, como el Modelo de Adopción de Comercio Electrónico (MACE) propuesto por Lee, Park y 
Ahn (2000) y el Modelo de Consumo Electrónico (MCE) propuesto por García (2007a).

Cabe destacar que existen otros modelos globales explicativos del consumo electrónico, más centrados en procesos cognitivos de la persona, entre ellos se pueden mencionar: el modelo de Nicosia (1966 citado por Herrero, 2005) que explica el proceso de toma de decisiones de consumo como una transición desde un estado pasivo a otro activo; modelo de Howard y Sheth (1969 citado por Herrero, 2005) propone tres niveles de aprendizaje en los consumidores para la toma de decisiones; modelo de comportamiento del consumidor de Engel, Kollat y Blackwell (1968, 1978 citado por Herrero, 2005) propone un proceso de cuatro componentes (estímulos, evaluación de información, variables ambientales y decisión de compra), el modelo de Bettman (1979 citado por Herrero, 2005) centrado en el procesamiento de la información, entendiendo la conducta de consumo como un proceso de selección. El modelo de Howard (1989 citado por Herrero, 2005) plantea la relación entre siete variables la información, el reconocimiento, la actitud, la confianza, la intención, la compra y la satisfacción de compra.

Sin embargo, el TAM ha sido ampliamente utilizado en la investigación sobre Internet y el comercio electrónico (Rodríguez del Bosque \& Herrero, 2008), este representa un modelo sencillo basado en las actitudes del consumidor (Herrero, 2005). Una adaptación de este modelo adecuado al contexto de habla hispana, se encuentra en el estudio de García (2007a) abordando la PU del comercio electrónico, partiendo de la consideración que la brecha económica se refleja también en una brecha digital entre países desarrollados y en vías de desarrollo, cuyo modelo se presenta en la (Figura 2).

García (2007a) plantea que las teorías de Valores Universales (Schwartz, 1992 citado en García, 2007b) y de la Motivación Humana (Maslow, 1943 citado en García, 2007b) explican las necesidades que llevan a los consumidores a adquirir determinados productos; la TAR (Ajzen \& Fishbein, 1980) explica el consumo en centros comerciales; la Teoría de la Conducta Planificada (Ajzen, 1985) explica el consumo en autoservicios, la Teoría de la Aceptación de la Tecnología (Davis, 1989) explica el consumo de computadoras personales y telefonía móvil MACE (Lee, Park \& Ahn, 2000) explica los riesgos que impiden la transacción digital y la Teoría Social Cognitiva (Bandura, 1987) explica la interacción entre el usuario y la red.

\section{FIGURA 2}

Modelo el Consumo Electrónico

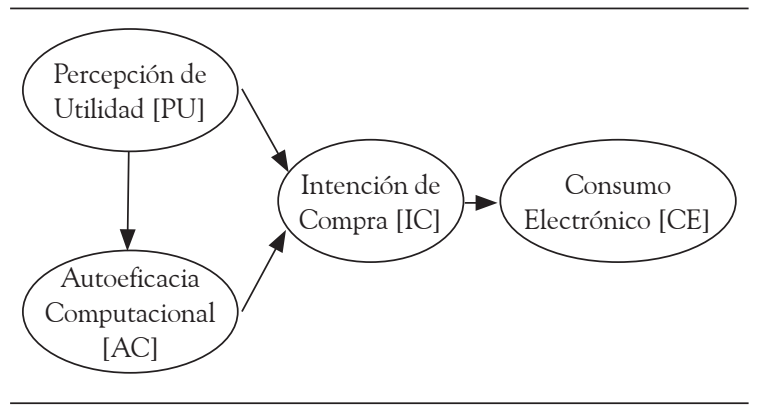

Fuente: García, 2007a.

García (2007a) en su MCE (Figura 2) plantea la interacción entre las siguientes variables: percepción de utilidad como variable latente exógena, autoeficacia computacional e intención de compra como variables latentes endógenas mediadoras y consumo electrónico como variable latente endógena.

El modelo indica como su primera trayectoria: $\mathrm{PU} \rightarrow \mathrm{IC} \rightarrow \mathrm{CE}$. Es decir, las personas evalúan, valoran y son impulsadas por beneficios que esperan obtener al comprar un producto o adquirir un servicio en Internet. Estos beneficios esperados son los que llevan a las personas a buscar y cotizar el precio del producto o servicio para finalmente adquirirlo.

La segunda trayectoria del MCE es: $\mathrm{PU} \rightarrow \mathrm{AC}$ $\rightarrow \mathrm{IC} \rightarrow \mathrm{CE}$. Indicando que serán las evaluaciones y valoraciones positivas de un producto o servicio las que activan necesidades en las personas, las cuales al suponer que tienen la habilidad para buscar y cotizar el precio del producto o servicio, terminaran realizando una transacción de compra en Internet.

A continuación se presentan las definiciones de cada una de las variables medidas en este estudio: 
Consumo electrónico (CE): aunque García (2006) considera el consumo electrónico como la transferencia electrónica de dinero en la compra de un producto o adquisición de un servicio; a efectos de esta investigación, se considerará consumo electrónico el uso de un bien económico que implica recibir algún tipo de beneficio; sin embargo, no necesariamente implica una retribución por el beneficio recibido, pues en el caso de bienes que se intercambian en Internet el beneficio de quien lo ofrece, muchas veces, es pagado por un tercero (anunciante).

Intención de Consumo (IC): Si bien el autor del MCE se refiere a la intención de compra, definiéndola como "la probabilidad de llevar a cabo una transferencia electrónica de dinero para comprar un producto o adquirir un servicio" (García, 2006, p. 4); a los efectos de esta investigación, se hará referencia a intención de consumo, y no intención de compra, como la probabilidad de hacer uso de un bien o servicio ofrecido a través de la vía electróni$\mathrm{ca}$, sin que esto necesariamente implique un pago.

La Autoeficacia Computacional (AC) se refiere a la confianza que posee una persona en sus habilidades para realizar con éxito una tarea relacionada con el computador (Marakas, Yi \& Johnson, 1998); este constructo, por ser fruto de autorreporte, representa la opinión de una persona acerca de su propia capacidad para utilizar la computadora y refleja determinadas capacidades para aplicar destrezas simples ante el computador, al momento de realizar a tareas complejas (Peinado, 2007), como las que se requieren para realizar consumos electrónicos.

La Percepción de Utilidad (PU): definida por García (2007ª , p. 416) "como la expectativa evaluativa, actitudinal y motivacional de obtener mayores beneficios y menores costos con respecto al uso o consumo de algún producto o servicio".

\section{Método}

\section{Descripción de la muestra}

El estudio, a través del cual se probaron las escalas del instrumento objeto de este reporte de investiga- ción, se realizó en una muestra de 217 estudiantes de posgrado en Gerencia de la Universidad Simón Bolívar; para obtener una condición similar a la de las muestras de estudiantes universitarios usadas por Lee, Park \& Ahn (2000) para su estudio de MACE y por García (2007a) para su MCE. Adicionalmente, también se consideró la intención de aumentar las probabilidades de que los informantes dispusieran de medios para consumir y/o adquirir bienes a través de Internet.

Se obtuvieron 214 cuestionarios válidos, puesto que los restantes 3 fueron invalidados por estar incompletos. Los informantes de género femenino corresponden al 59,8 \% de la muestra mientras que el restante $40,2 \%$ son de género masculino; sus edades estuvieron comprendidas entre los 22 y los 68 años, con $M=30,68$ años y $D S=$ 6,421 .

\section{TABLA 1}

Distribución por profesión

\begin{tabular}{lcc}
\hline \multicolumn{1}{c}{ Profesión } & $f$ & $P$ \\
\hline Ciencias del Agro y del Mar & 1 & 0,47 \\
Ciencias de la Educación & 1 & 0,47 \\
Humanidades, Letras y Artes & 1 & 0,47 \\
Ciencias Básicas & 3 & 1,40 \\
Ciencias de la Salud & 5 & 2,34 \\
Ingeniería, Arquitectura y & 63 & 29,44 \\
Tecnología & 83 & 38,79 \\
Ciencias Sociales & 57 & 26,64 \\
Sin Clasificación & 214 & 100 \\
Total &
\end{tabular}

Fuente: elaboración propia.

La distribución de la muestra por profesión y posgrado se ilustra en las Tablas 1 y 2 donde se evidencia que, la mayoría de los informantes pertenecen al área de las Ciencias Sociales, Ingeniería, Arquitectura y Tecnología cursantes de posgrado en Especialización de Gerencia de Empresas. 
TABLA 2

Distribución por posgrado que cursa

\begin{tabular}{lll}
\hline \multicolumn{1}{c}{ Postgrado } & $f$ & \multicolumn{1}{c}{$P$} \\
\hline Maestría & 1 & 0,50 \\
Psicología & 13 & 6,10 \\
Sistemas de información & & \\
Especialización & 12 & 5,60 \\
Gerencia en Telecomunicaciones & 12 & 5,60 \\
Auditoria de Estado & 14 & 6,50 \\
Gerencia de tecnología & 16 & 7,50 \\
Finanzas & 19 & 8,90 \\
Desarrollo Organizacional & 22 & 10,30 \\
Gerencia en mercadeo & 44 & 20,60 \\
Gerencia de proyectos & 59 & 27,60 \\
Gerencia de empresas & 2 & 0,90 \\
No contestó & 214 & 100 \\
Total &
\end{tabular}

Fuente: elaboración propia.

La distribución de la muestra por ocupación no se reporta, debido a que se obtuvieron 80 categorías de respuestas muy heterogéneas que no permitieron obtener clasificaciones relevantes.

TABLA 3

Distribución por nivel socioeconómico

\begin{tabular}{ccc}
\hline Nivel socioeconómico & $f$ & $P$ \\
\hline 1 & 0 & 0 \\
2 & 0 & 0 \\
3 & 1 & 0,5 \\
4 & 18 & 8,4 \\
5 & 48 & 22,4 \\
6 & 66 & 30,8 \\
7 & 56 & 26,2 \\
8 & 11 & 5,1
\end{tabular}

\begin{tabular}{lll}
\hline \multicolumn{1}{c}{ Nivel socioeconómico } & & \multicolumn{1}{c}{$P$} \\
\hline 9 & 2 &, 9 \\
10 & 0 & 0 \\
No contestó & 12 & 5,6 \\
Total & 214 & 100 \\
\hline
\end{tabular}

Fuente: elaboración propia.

La distribución de la muestra según el nivel socioeconómico concentra un 79,8 \% de los informantes en los estratos medios 5, 6 y 7; mientras que los extremos 1 y 10 no fueron seleccionados por ningún informante.

\section{Procedimiento}

Para obtener las escalas de medición de IC, AC, $\mathrm{PU}$ y $\mathrm{CE}$, que componen el instrumento evaluado en este estudio:

1. Se solicitó al autor del MCE, el acceso al formato completo del instrumento, así como la autorización para hacer uso del mismo. Dicho instrumento incluye cuatro escalas correspondientes a cada una de las variables de MCE (C. García, comunicación personal, 5 de marzo de 2008).

2. Los investigadores verificaron la comprensión de contenido de los ítems de las escalas de dicho instrumento a través de una revisión de la terminología, esto permitió ajustar la redacción general del instrumento y justificó la inclusión de instrucciones, que fueron separadas de la presentación enmarcándolas en un recuadro.

3. Se modificaron las referencias específicas al contexto original para el cual fue diseñado el instrumento (el nombre de las divisiones de la universidad mexicana donde se aplicó; así como algunos sitios de Internet de uso local, referencia a información de frontera, etc.).

4. Se decidió eliminar la referencia a la comunidad científica, al consumo erótico o de otros bienes de consumo en particular; ya que no se considera conveniente hacer referencia a categorías específicas de bienes o usuarios, cuyos patrones 
de consumo podrían ser determinados por las características del bien o servicio y no por el medio electrónico a través del cual se realiza; además de constituir elementos sensibles o no aplicables, que pudieran aumentar la ausencia de respuestas.

5. Con la intención de aumentar la generalización se introdujeron referencias a la afiliación a membresías, se eliminaron las referencias al tipo de medio de transporte de viajes y se sustituyó la referencia a conciertos por la de espectáculos, considerada más amplia.

6. Con el fin de actualizar las tendencias de consumo se añadieron referencias a la descarga de imágenes, melodías, videos y software, así como a servicios bancarios y de telefonía.

7. Se decidió incluir en la escala IC, un ítem relacionado con el uso de la tarjeta de crédito, para identificar una eventual dificultad con su uso, comúnmente usado para compras en línea.

8. El cuestionario adaptado, incluye información demográfica de los informantes: el género, nivel socio-económico; la edad, la profesión, la ocupación, y tipo de posgrado en curso.

9. Se solicitó la evaluación del contenido y forma del cuestionario por parte de tres expertos en las áreas de Economía, Psicología y metodología; con la finalidad de comprobar que se mantuviera el sentido de los constructos medidos por las escalas, posteriormente algunas observaciones hechas por estos expertos fueron incorporadas. Como resultado del procedimiento anterior, se realizaron las siguientes modificaciones al instrumento propuesto:

a. Se agregaron nuevos sitios de Internet de uso común en Venezuela (www: amazon. com, tucarro.com, porlapuerta.com, tucine.com, mercadolibre.com y ebay.com), así como preguntas abiertas y espacios para comentarios.

b. Se cambió la escala de respuestas del instrumento de intención de consumo (de muy improbable, imposible, posible, muy probable) a una escala que expresa sólo probabilidad de ocurrencia (muy probable, probable, poco probable e improbable); debido a que los ex- pertos coincidieron en indicar que la escala inicial de respuesta era confusa debido a que la intención debe diferenciarse de la posibilidad de realizar la acción.

c. Se cambió la escala de respuestas del instrumento de percepción de utilidad (de totalmente en desacuerdo, en desacuerdo, de acuerdo, totalmente de acuerdo) a una escala que expresa grado de utilidad (muy útil, útil, poco útil e inútil). Este ajuste dio respuesta a las sugerencias de los expertos en relación a que la escala original de respuesta era incoherente con la redacción de los ítems que pretendían medir el constructo.

d. Se modificaron las opciones de respuesta de la escala consumo electrónico a opciones que se expresan en rangos de ocurrencia mensual (de 1 a 9 veces, de 10 a 19 veces, de 20 a 29 veces, 30 o más veces).

e. Se modificaron algunos términos en la redacción de las preguntas abiertas y se incluyeron alternativas en las opciones de medios de pago de la sección $\mathrm{N}^{\circ} 6$ del IVACE (Apéndice).

f. Se agregó una columna de respuesta en la que podía indicarse el carácter gratuito o remunerado del consumo asociado al ítem, en dos versiones de posicionamiento: antes del ítem o después de la escala original de respuesta.

g. Se decidió cambiar el orden en que se muestran las escalas, presentando primero IC y por último $\mathrm{CE}$; de manera que, alejando ambas escalas, las respuestas de la primera generaran sesgo producto de coherencia forzada, que pudiera inducir la respuesta de testiguar que realizó el consumo que antes había afirmado tener intenciones de consumir por no contradecirse, antes que por veracidad.

\section{Instrumento de recolección de datos}

El instrumento resultante de esta primera fase de adaptación y diseño, constituye una versión diferente del instrumento usado por García (2007a); 
pues, aún cuando se mantuvo el contenido de una parte de los ítems de sus escalas, la redacción de los mismos fue modificada, así como las opciones de respuestas de tres de las cuatro escalas originalmente propuestas. Este nuevo instrumento será llamado Inventario de Variables Asociadas al Consumo Electrónico (IVACE) y pretende medir las variables IC, AC, PU y CE a través de escalas de Likert diseñadas para cada una de dichas variables, que pondera las respuestas dadas por los informantes.

\section{Análisis y discusión de resultados}

A continuación se presentan en dos apartados, los resultados obtenidos de los datos recolectados y su análisis. En primer lugar, se presentan los resultados descriptivos de las características sociodemográficas, económicas y académicas de la muestra, así como algunos aspectos de sus prácticas de consumo electrónico, relacionadas con los sitios y las formas de pago usados del IVACE ( $\mathrm{N}^{\circ} 3$, 4, 5, 6, 7 y 8). En segundo lugar, se presenta el análisis psicométrico de cada una de las escalas de medición de variables incluidas en el IVACE ( ${ }^{\circ} 9,10,11$ y 12$)$.

\section{Análisis descriptivo}

En la sección $\mathrm{N}^{\circ} 3$ de descripción académicoprofesional de la muestra, IVACE (Apéndice), los ítems abiertos profesión (Tabla 1) y ocupación generaron clasificaciones heterogéneas. En profesión, se reagruparon en categorías preestablecidas, escogiéndose a tal fin las nomenclaturas de carreras por área de conocimiento descritas por el Consejo Nacional de Universidades, Oficina de Planificación del Sector Universitario (2003). Se creó la categoría Sin Clasificación (Tabla 1) para agrupar las respuestas que no permitían ubicar al informante en una categoría específica.

El ítem ocupación, no se reportó puesto que la diversidad de las respuestas y ambigüedad de algunas de ellas, no permitió establecer una clasificación relevante.

Para el ítem postgrado (Tabla 2) se obtuvieron categorías previsibles: los posgrados propios de la institución de la cual procedían los informantes; apenas un participante pertenecía a un área diferente a los de la Coordinación de Gerencia.

Al solicitar información sobre los sitios de Internet más usados $\mathrm{N}^{\circ} 5$ Apéndice, se pudieron clasificar dichos sitios, distinguiendo entre los que son visitados por el mayor número de informantes. Como se ilustra en la Figura 3, el 94,4 \% de la muestra usa la página del motor de búsqueda Google, seguida por Hotmail; mientras que otros sitios preestablecidos como AOL (0,9 \%) y Latinmail (1,4 $\%)$ son utilizados por menos del $2 \%$ de la muestra.

Adicionalmente se ofrecía una opción abierta para agregar sitios de Internet, por esa vía se obtuvieron los nombres de otros cincuenta y ocho sitios web que fueron agrupados y aparecen en la Figura 3 como "Otros". El resultado de esta clasificación establece que el $62 \%$ de la muestra usa sitios web que no están en la lista preestablecida del IVACE; sin embargo, de los sitios web sugeridos sólo tres superan el 5 \%: Facebook (9,8 \%), Gmail (5,6\%) y Noticias 24 (5,1\%).

Para la sección correspondiente a medio de pago $\mathrm{N}^{\circ} 6$ del IVACE Apéndice, se puede observar que la mayor parte de la muestra dispone de la Tarjeta de Crédito (TC) (92,1\%) para consumo electrónico. Este alto porcentaje pudiera estar relacionado con las restricciones (Normativa) establecidas por el control cambiario venezolano (CADIVI), que sólo aprueba este medio para compras a través de Internet.

\section{TABLA 4}

Medios de Pago disponibles para el consumo electrónico

\begin{tabular}{lcc}
\hline \multicolumn{1}{c}{ Medio de pago } & $F$ & $P$ \\
\hline Tarjeta de crédito & 197 & 92,1 \\
Transferencia bancaria & 75 & 35 \\
Depósito bancario & 40 & 18,7 \\
E-Card & 15 & 7,0 \\
Gift-Card & 15 & 7,0 \\
Otros: Pay-Pal & 2 & 0,9 \\
\hline
\end{tabular}

Fuente: elaboración propia. 
FIGURA 3

Sitios de Internet usados por la muestra.

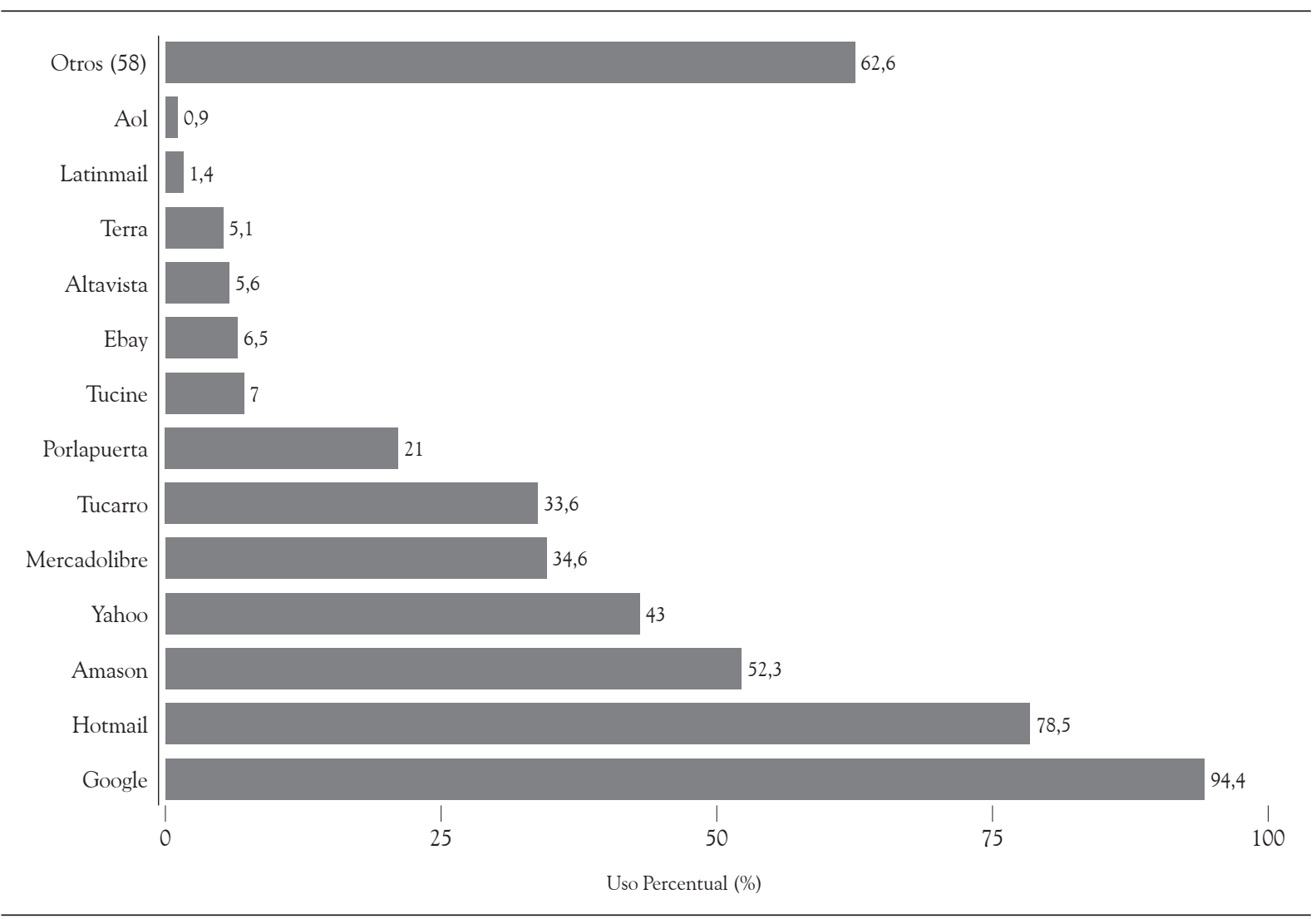

Fuente: elaboración propia

En cuanto al análisis descriptivo de la disponibilidad de TC habilitada para la compra por Internet ( $\mathrm{N}^{\circ} 7$ IVACE, Apéndice), los hallazgos señalan que el $88,3 \%$ de la muestra la tiene habilitada mientras que $11,7 \%$ no. Se puede destacar que de los 197 sujetos que reportaron contar con TC para consumo electrónico (Tabla 4) solo 189 reportaron tenerla habilitada, esta diferencia puede deberse a que no poseen tarjeta de crédito o tienen tarjetas prepago (cuyo uso no es permitido por el control cambiario).

En relación a las dificultades asociadas a la compra por Internet ( $\mathrm{N}^{\circ}$ 8-Apéndice) los resultados indican que el $67,8 \%$ de la muestra reportaron no haber tenido dificultades mientras que el 32,2\% sí. Para evaluar estas dificultades, se realizó un análisis de contenido de la pregunta abierta asociada al ítem que permitió identificar cuatro categorías cuya descripción se presenta a continuación:

Dificultades relacionadas con el proceso de compra: representan el 18,31 \% de las dificultades reportadas y están relacionadas con los tiempos de respuesta o procesamiento de la solicitud, poca amigabilidad de los sitios, por el idioma del mismo o lo complicado de los procesos de adquisición de los productos o servicios; así como la inconformidad con las características del producto que se recibe y la imposibilidad de usar determinados medios de pago, como la TC.

Dificultades relacionadas con el envío: representan el 14,08 \% de las dificultades reportadas asociadas a los obstáculos o malas experiencias como perdidas, daños o retrasos durante el envío de productos, así como requisitos engorrosos para el envío y disponibilidad de casilleros. 
Dificultades relacionadas con la desconfianza e inseguridad: representan el 21,13\% de las dificultades reportadas, debidas al temor de estafas o pérdidas financieras y de violación de la privacidad o divulgación indebida de datos personales.

Dificultades relacionadas con el control cambiario: representan el 46,48 \% de las dificultades reportadas y son propias del contexto socioeconómico de la muestra; debido a que desde el 2003, en Venezuela se mantiene un control cambiario que limita las sumas y los medios para compras electrónicas en el exterior. De modo que se necesita la aprobación de los medios de pago por parte de la instancia gubernamental que regula la compra en moneda extranjera, dichos medios se limitan al uso de la tarjeta de crédito y el monto permitido para la compra ha ido disminuyendo hasta establecerse en 400 USD anuales por persona, a partir del $1^{\circ}$ de enero 2009.

En cuanto a la columna de respuesta que solicitaba indicar el carácter gratuito o pago de la actividad a la que se refería cada ítem, los hallazgos no permiten reportar resultados concluyentes ya que, independientemente de la posición donde se encontraba la columna, la mayor parte de los informantes dejaron de responderla, esto sugiere una dificultad de comprensión generalizada.

\section{Análisis psicométrico de las escalas del IVACE}

Para estudiar la validez de constructo del IVACE se realizó un análisis factorial de componentes principales con rotación Varimax y criterio Kaiser, dicho estadístico permitió obtener el porcentaje de la varianza total explicada por cada una de las escalas y subescalas o factores del mismo. Para el análisis factorial de cada escala, se consideraron sólo los ítems cuya carga factorial fue mayor a .40 y cargaron exclusivamente en un factor.

La confiabilidad fue medida a través del índice de consistencia interna Alfa de Cronbach $(\propto)$, que fue calculado para cada escala del IVACE, así como para cada una de las subescalas.

\section{Intención de Consumo}

El análisis factorial, reporta un porcentaje de la varianza total explicada de la escala de 47,32\%, para el Factor 1 de 30,41 \% y para el Factor 2 de $16,91 \%$.

Se eliminaron los ítems relacionados con consumo de bienes con características particulares, identificados con los adjetivos: clandestinos, piratas y esotéricos ítems 4, 5 y 6 (ANEXO), puesto que parecieran generar sesgo asociado a la deseabilidad social y no están presentes en las otras escalas que identifican variables del MCE. En el análisis factorial estos ítems se habían agrupado en el Factor 2 de la escala Intención de Consumo, esto ocasionó que esta subescala se descartada. Por tanto, el Factor 1 quedo conformado como se muestra en la Tabla 5.

Esta escala se puede definir como la probabilidad de hacer uso de un bien o servicio ofrecido a través de la vía electrónica, sin que esto necesariamente implique un pago.

\section{TABLA 5}

Análisis factorial de componentes principales de la Escala Intención de Consumo

\begin{tabular}{clc}
\hline & \multicolumn{1}{c}{ Ítems } & $\begin{array}{c}\text { Intención } \\
\text { de consumo } \\
\propto\end{array}$ \\
\hline 1 & Compraría los productos en oferta & 0,536 \\
2 & Contrataría los servicios en oferta & 0,552 \\
3 & Adquiriría información en oferta & 0,472 \\
7 & Compraría productos de prestigio & 0,700 \\
8 & Contrataría servicios de calidad & 0,796 \\
9 & Adquiriría información relevante & 0,589 \\
10 & Compraría productos de moda & 0,691 \\
11 & Contrataría servicios actualizados & 0,822 \\
12 & Autorizaría mi tarjeta de crédito & 0,431 \\
\hline
\end{tabular}

Fuente: elaboración propia.

La confiabilidad de esta escala resultó $\propto=$ 0.809 , lo que sugiere un alto nivel de confiabilidad y supera ligeramente los resultados reportados por 
García, quien reportó $\propto=.780$ (C. García, comunicación personal, 5 de marzo de 2008).

En el análisis de contenido de las respuestas a la pregunta ¿Qué otro tipo de operaciones tendría usted intención de realizar a través de alguno de estos sitios? se identificaron cinco categorías de operaciones reportadas por los informantes, a saber: entretenimiento, venta (publicación de ofertas), pago u operaciones bancarias (transferencias/ pagos, manejo cuentas extranjera, manejo tarjetas de crédito), servicios (reservaciones, afiliaciones, búsqueda de información, financieros, recreativos y cursos en línea, trámites burocráticos, búsqueda de trabajo) y compra (tecnología, equipos, servicios, productos importados o escasos, inmuebles, boletos software, libros electrónicos, videos, electrodomésticos, etc.)

\section{Autoeficacia Computacional}

El porcentaje de la varianza total explicada de la escala fue de $67,42 \%$, el Factor 1, autoeficacia reactivo explica el 44,38 \% y el Factor 2, autoeficacia proactiva explica el 23,03 \% de la varianza.

De acuerdo al resultado obtenido en el análisis se propone eliminar el ítem 3 (que cargó en el fac- tor ACR), debido a que al observar su redacción resulta poco coherente con el contenido de los otros dos ítems 1 y 2 que cargan en el mismo factor. Este resultado puede estar asociado al hecho de que el ítem en cuestión, hace referencia a la búsqueda de información científica y sus respuestas pueden estar sesgadas por el efecto de la deseabilidad social, debido a que la aplicación del cuestionario se realizó en un entorno académico (Tabla 6).

Los factores de la escala puede ser definidos como: (a) ACP: la confianza que tiene una persona de sus habilidades para realizar una acción que modifique su condición actual (inscribirse, seleccionar, comprar, contratar, bajar y encontrar) a través de los sitios web utilizados, (b) ACR: la confianza que tiene una persona de sus habilidades para evitar una acción indeseada (eliminar y evitar) a través de los sitios web utilizados.

La confiabilidad de la escala de Autoeficacia Computacional fue de $\propto=0.869$ este resultado es similar a los hallados por García, que obtuvo $\propto=0.900$ (C. García, comunicación personal, 5 de marzo de 2008). Para la subescala ACP de (7 ítems) fue de $\propto=0.920$ y para la de ACR ( 2 ítems) fue de $\propto=0.711$, lo que sugiere altos niveles de confiabilidad.

\section{TABLA 6}

Análisis factorial de componentes principales de la Escala Autoeficacia

\begin{tabular}{llcc}
\hline \multicolumn{1}{c}{ Items } & $\begin{array}{c}\text { Autoeficacia } \\
\text { Computacional Proactiva } \\
\propto=.920\end{array}$ & $\begin{array}{c}\text { Autoeficacia } \\
\text { Computacional Reactivo } \\
\propto=.711\end{array}$ \\
\hline 1 & Eliminar las ofertas inesperadas & & 0,866 \\
2 & Evitar la publicidad repentina & 0,774 \\
4 & Seleccionar ofertas que necesito & 0,612 & \\
5 & Elegir con quien chatear & 0,697 & \\
6 & Inscribirme en cursos y membresías que necesito & 0,795 & \\
7 & Comprar los productos que busco & 0,868 & \\
8 & Contratar los servicios que me gustan & 0,848 & \\
9 & Encontrar ofertas especiales & 0,871 & \\
10 & Bajar imágenes, sonidos y videos & 0,761 & \\
\hline
\end{tabular}

Fuente: elaboración propia. 
A través del análisis de la pregunta ¿Qué otro tipo de operaciones cree usted saber hacer en alguno de estos sitios? se identificaron otras categorías de habilidades autorreportadas, tales como: realizar transacciones bancarias, controlar spam, configurar perfil de usuario, participar en foros, ver, descargar o publicar información-videos-películas-música. Estas habilidades parecieran reafirmar que prevalece el factor autoeficacia proactiva identificado en la escala.

\section{Percepción de utilidad}

El porcentaje de la varianza total explicada de la escala fue de 54,93\%, el Factor 1, Percepción de Utilidad de Consumo Electrónico explica el 28,87 \% y el Factor 2, Percepción de Utilidad de Mejoramiento de Habilidades explica el 26,07 \% de la varianza. La Tabla 7 muestra la carga factorial de los ítems de ambas subescalas, y se definen como: (a) Percepción de Utilidad de Consumo Electrónico, es la expectativa evaluativa, actitudinal y motivacional de obtener mayores beneficios y menores costos con respecto al uso o consumo de algún producto o servicio electrónico (García, 2007ä, p. 416), y (b) Percepción de Utilidad de Mejoramiento de Habilidades, es la expectativa evaluativa, actitudinal y motivacional de obtener mayores beneficios y menores costos con respecto al uso o consumo de algún producto o servicio (García, 2007ª , p. 416) que permitan mejorar las destrezas para el manejo de sistemas electrónicos de consumo.

La confiabilidad fue de $\propto=0.849$, este resultado es similar a los hallados por García, que obtuvo $\propto=0.880$ (C. García, comunicación personal, 5 de marzo de 2008), mientras que la sub escala Percepción de Utilidad de Consumo de (5 ítems) fue de $\propto=0.818$ y para Percepción de Utilidad de Mejoramiento de Habilidades (5 ítems) fue de $\propto=$ 0.718 , lo que sugiere altos niveles de confiabilidad.

Al explorar con una pregunta abierta acerca de otras razones para considerar útil el uso de Internet, los informantes identificaron cualidades tales como la rapidez, la comodidad, la facilidad, la eficiencia, la disponibilidad de información u orientación y la conformación de redes, como dimensiones de la utilidad percibida.

TABLA 7

Análisis factorial de componentes principales de la Escala Percepción de Utilidad

\begin{tabular}{llcc}
\hline \multicolumn{1}{c}{ Ítems } & $\begin{array}{c}\text { Percepción de utilidad } \\
\text { de consumo electrónico } \\
\propto=0.818\end{array}$ & $\begin{array}{c}\text { Percepción de utilidad } \\
\text { de mejoramiento de } \\
\text { habilidades } \propto=0.718\end{array}$ \\
\hline 1 & Se compran las cosas que uno busca & 0,419 & \\
4 & Se contratan los servicios que uno busque & 0,662 & \\
8 & Se compra fácilmente & 0,825 & \\
9 & Se contratan servicios fácilmente & 0,872 & 0,666 \\
10 & Se hacen fácilmente inscripciones a cursos /membresías & 0,710 & 0,530 \\
2 & Se chatea con el tipo de personas que uno busque & & 0,564 \\
3 & Se consulta información académica de interés & & 0,661 \\
5 & Se mejoraran los criterios de compra & & 0,805 \\
6 & Se desarrollan habilidades comunicativas & & \\
7 & Se aprende a encontrar cualquier tipo de información & & \\
\hline
\end{tabular}

Fuente: elaboración propia. 


\section{Consumo electrónico}

El análisis factorial de la escala mostró un sólo factor llamado Consumo Electrónico, por tanto, no se puede presentar los resultados del método de rotación Normalizada Varimax con Kaiser. En la Tabla 8 se presentan los resultados del análisis de extracción de componente principal, sin la normalización Varimax con criterio Kaiser. Se eliminó el ítem 3, por la baja carga factorial.

TABLA 8

Análisis factorial de componentes principales de la Escala Consumo Electrónico

\begin{tabular}{|c|c|c|}
\hline \multicolumn{2}{|r|}{ Ítems } & \multirow{2}{*}{$\begin{array}{c}\begin{array}{c}\text { Consumo } \\
\text { electrónico } \\
\propto=0.866\end{array} \\
0,660\end{array}$} \\
\hline 1 & $\begin{array}{l}\text { He comprado videos, imágenes, } \\
\text { películas }\end{array}$ & \\
\hline 2 & He contratado servicios & 0,675 \\
\hline 4 & $\begin{array}{l}\text { He comprado melodías, ringtones o } \\
\text { música }\end{array}$ & 0,711 \\
\hline 5 & He contratado servicios de telefonía & 0,706 \\
\hline 6 & En estos sitios he comprado libros & 0,754 \\
\hline 7 & $\begin{array}{l}\text { En estos sitios he adquirido boletos } \\
\text { para espectáculos }\end{array}$ & 0,780 \\
\hline 8 & $\begin{array}{l}\text { En estos sitios he adquirido hard- } \\
\text { ware /software }\end{array}$ & 0,693 \\
\hline 9 & He comprado boletos para viajar & 0,725 \\
\hline 10 & He contratado servicios bancarios & 0,503 \\
\hline 11 & $\begin{array}{l}\text { Me he inscrito en cursos / membre- } \\
\text { sías }\end{array}$ & 0,680 \\
\hline
\end{tabular}

Fuente: elaboración propia.

Esta escala de consumo electrónico se puede definir como el uso de un bien económico que implica recibir algún tipo de beneficio.

En cuanto a la confiabilidad de la escala resultó de $\propto=0.866$, lo que sugiere altos niveles de confiabilidad, es ligeramente mayor a los reportados por García (C. García, comunicación personal, 5 de marzo de 2008), que obtuvo un $\propto=0.800$.
En el análisis de contenido de las respuestas a la pregunta ¿Qué otro tipo de operaciones ha realizado a través de alguno de estos sitios? se identificaron cinco categorías equivalentes a las identificadas en el análisis de la pregunta abierta sobre intención de consumo, a saber: entretenimiento, venta, pago u operaciones bancarias, servicios y compra.

\section{Conclusiones y recomendaciones}

El análisis de la validez y confiabilidad del IVACE permitió establecer que las cuatro escalas de medición de variables asociadas al consumo electrónico poseen altos niveles de confiablidad, superando todas ellas el valor de $\propto=0.80$.

El análisis factorial evidenció que las escalas de IC y CE tienen una dimensión única, que sugiere consistencia de las variables medidas en una estructura bien delimitada; para estas dos escalas se identificaron además categorías adicionales de intenciones o consumo que resultaron equivalentes, por lo que pareciera corroborarse un estrecho nexo entre la intención y la acción de consumo electrónico, tal y como lo plantea el MCE.

La escala de AC reveló dos dimensiones: Autoeficacia Computacional Proactiva y Autoeficacia Computacional Reactiva. Sin embargo, la dimensión que agrupa mayor número de ítems es la proactiva, a la que también parecieran corresponder las categorías de habilidades que se identificaron a partir de las respuestas a la pregunta abierta correspondiente a dicha escala.

La escala de PU resultó compuesta por dos dimensiones: la Utilidad de Consumo Electrónico propiamente dicha y la Utilidad asociada al Mejoramiento de Habilidades, revelando así que el medio electrónico ofrece beneficios que están asociados al uso del medio y no necesariamente al fin de consumo para el cual se usa dicho medio. Adicionalmente, el análisis de contenido permitió identificar elementos que los informantes reconocen como de utilidad (rapidez, comodidad, facilidad, eficiencia, disponibilidad de información u orientación y conformación de redes). Dichos elementos parecieran reflejar la variable que Da- 
vis (1989) en su modelo denomina Percepción de Facilidad de Uso, definida como la creencia asociada a que el uso de un sistema requiere menor esfuerzo, y cuya inclusión podría plantearse en una modificación del IVACE.

El análisis de los elementos de forma del IVACE que sugieren modificaciones o adaptaciones para mejorar su eficacia en el entorno local se presentan a continuación.

La información demográfica obtenida a través de ítems abiertos conduce a categorías de respuestas muy heterogéneas de difícil manejo. Mientras que la información académica obtenida con ese mismo tipo de preguntas resulta previsible, ya que enumera los postgrados propios de la coordinación a la cual pertenecían los informantes de la muestra.

La mayoría de los sitios web enumerados en el instrumento resultaron ser de uso frecuente para los informantes, sólo dos de ellos (Latinmail y Aol) son usados por menos del $2 \%$ de la muestra. De igual forma, los sitios web adicionalmente sugeridos por los informantes son tres (Facebook, Gmail y Noticias 24) que son usados por más del $5 \%$ de los informantes.

Los resultados parecieran indicar que el establecimiento de un control cambiario y su modalidad de ejecución (que establece un cambio fijo de la tasa de cambio bolívar/dólar, la restricción del gasto en moneda extranjera a montos fijos anuales que deben distribuirse entre consumos realizados en el exterior y consumos realizados por Internet) determina que en el caso de estudio, el medio de pago prevaleciente para el consumo electrónico sea el de la TC.

Ahora bien, aunque el control cambiario es considerado como dificultad al momento de realizar compras por Internet, el hecho de que el 88,3\% de la muestra tenga habilitada su TC para realizar dichas transacciones, pareciera significar que la dificultad no reside en la obtención o habilitación del medio de pago, sino en el límite fijado al gasto máximo de la compra.

La desconfianza e inseguridad (21,13\%) es considerada la segunda dificultad al momento de realizar compras por Internet. En general todas las dificultades mencionadas anteriormente parecen guardar cierta correspondencia con los elementos asociados a la percepción de riesgo mencionados por Lee, Park \& Ahn (2000) quienes, en su modelo de Riesgo Percibido, consideran los riesgos asociados a los productos o servicios y aquellos al contexto de la transacción, entre estos últimos: privacidad, seguridad (autenticación), no incumplimiento y en general riesgo percibido en transacciones en línea.

El análisis y las conclusiones establecidas en esta investigación conducen a plantear las siguientes recomendaciones para mejorar la eficacia del IVACE:

- Modificar el formato de respuesta de los profesión, ocupación y posgrado que cursan los informantes, por respuestas cerradas de selección simple a escoger dentro de categorías estándar preestablecido según el contexto.

- Establecer como porcentaje mínimo el $5 \%$ de uso entre la muestra para excluir los sitios web predeterminadas e incluir las sugeridas. De esta manera, en el contexto evaluado se excluirían Latinmail y Aol incluyéndose Facebook, Gmail y Noticias 24.

- Diversificar la redacción de los ítems de la intención y consumo agregando actividades que no tengan necesariamente carácter remunerativo, por ejemplo: realizar transacciones bancarias.

- Incluir ítems relacionados con las categorías de intención y consumo sugeridas por la muestra.

- Incluir ítems que indaguen sobre aspectos más específicos de la utilidad percibidos.

- Incluir ítems de autoeficacia proactiva que reflejen las habilidades sugeridas por los informantes de esta muestra y/o de autoeficacia reactiva que reflejen el elemento inseguridad y desconfianza (ser capaz de evitar fraudes o establecer claves de acceso o certificados, por ejemplo).

- Eliminar la columna que señala el carácter gratuito o pago de la transacción, ya que la definición adoptada para el presente trabajo implica recibir algún tipo de beneficio, sin importar el tipo de retribución. 
- En la escala de Intención de Consumo se sugiere agregar "realizar transacciones, además de adquirir, contratar"; es decir tipos de consumo que no impliquen pago directo.

- En la escala de autoeficacia es necesario modificar las opciones de respuesta, pues dificulta la comprensión de los ítems.

- En la escala de la Utilidad Percibida se sugiere "agregar ítems que hagan referencias a los aspectos de la utilidad nuevo" que fueron sugeridos en el análisis.

- En la escala de consumo es necesario agregar "nunca" o frecuencia "O", en las opciones de respuesta.

En función de las mejoras planteadas en estas conclusiones, se sugiere realizar una nueva validación del IVACE una vez incorporadas las mismas. Así como, realizar estudios comparativos de otros modelos de comportamiento del consumidor electrónico alternativos al modelo evaluado en este estudio, que puedan adaptarse a las características del contexto venezolano.

\section{Referencias}

Ajzen, I. (1985). From intention to actions: A theory of planned behavior. In J. Kuhl \& J. Beckman (Eds.), Action control: From cognitions to behavior (pp. 11. 39). New York: Springer- Verlag.

Ajzen, I. \& Fishbein, M. (1980). Understanding attitudes and predicting social behavior. Englewood Cliffs, NJ: Prentice-Hall.

Bandura, A. (1987). Pensamiento y Acción. Fundamentos Sociales. España: Ediciones Martínez Roca.

Consejo Nacional de Universidades, Oficina de Planificación del Sector Universitario. (2003). Libro de Oportunidades de Estudio. Extraído el 9 de marzo de 2009, de http://loe.cnu.gov.ve/home.php

Cox, D. \& Rich, S. (1964). Perceived risk and consumer decision making: The case of telephone shopping. Journal of Marketing Research, 32-39.

Davis, F. (1989). Perceived usefulness, perceived ease of use, and user acceptance of information technology. MIS Quarterly, 13(3), 19-340.
Davis, F. \& Venkatesh, V. (1995). Measuring user acceptance of emerging information technologies: An assessment of possible methods biases. Proceedings of the $28^{\text {th }}$ Annual Hawaii International Conference on System Sciences, 729-736.

EFE. (2008a, mayo 28). Crecen los negocios por la web en Latinoamérica, destaca directivo de Google. Empresa Exterior. Extraído el 15 de julio, 2008, de http://www.empresaexterior.com/conte/14445.asp

EFE. (2008b, junio 25). Comercio electrónico creció un $40 \%$ en América Latina y el Caribe en 2007. Empresa Exterior. Extraído el 15 de julio, 2008, de http://www.empresaexterior.com/conte/15238.asp

García, C. (2007a). Percepción de la utilidad del comercio electrónico. Enseñanza e Investigación en Psicología, 2(2), 409-420.

García, C. (2007b). El consumo móvil de las generaciones futuras. Revista Universitaria Digital, 8 (5). Disponible en http://www.revista.unam.mx/vol.8/num5/ art36/int36.htm

García, C. (2006). Un modelo de consumo electrónico. AHCIET Revista de Telecomunicaciones. Nro. 108. Extraído el 5 de marzo, 2008, de http://www.ahciet.net/actualidad/revista/r. aspx?ids $=10664 \&$ ids $2=21748$

Gómez, A. (2004). La Web, todo en un portal. Rev. PC World Vzla., 46, 23-30.

Herrero, A. (2005). El proceso de adopción de nuevos sistemas de venta: aplicación al comercio al comercio electrónico entre particulares y empresas. Tesis de Doctorado, Universidad de Cantabria, Santander, España.

Iriarte, A. E. (s. f.). Comercio electrónico en América Latina: realidades y perspectivas. Extraído el 15 de julio, 2008, de http://lac.derechos.apc.org/investigación/comercio_electronico.doc

Lee, D., Park, J. \& Ahn, J. (2000, December). On the explanation of factors affecting e-commerce adoption. Twenty Second International Conference on Information Systems, New Orleans, Louisiana, USA.

Lederer, A., Maupin, D., Sena, M. \& Zhuang, Y. (2000). The Technology Acceptance Model and the World Wide Web. Decision Support Systems, 29, 269- 282.

Marakas, G., Yi, M. \& Johnson, R. (1998). The multilevel and multifaceted character of computer self-efficacy: Toward a clarification of the cons- 
truct and an integrative framework for research. Information Systems Research, 9(2), 126-163.

Master-Net.net (2007, noviembre). Situación del comercio electrónico en América Latina. Master-Net.net. ISSN 1576-9003. Extraído el 15 de julio, 2008, de http://www.masterdisseny.com/master-net/atrasadas/40.php3

Peinado, S. (2007). Efecto de los estilos de aprendizaje y la autoeficacia computacional sobre el desempeño en foro electrónico. Trabajo de Grado de Maestría, Universidad Simón Bolívar, Caracas, Venezuela.
Rodríguez del Bosque, I. A. \& Herrero, A. (2008). Antecedentes de la utilidad de percibida en la adopción del comercio entre particulares y empresas. Cuadernos de Economía y Dirección de la Empresa, 34, 107-134.

Teo, T., Lim, V. \& Lai, R. (1999). Intrinsic and extrinsic motivation in Internet usage. Omega, The International Journal of Management Science, 27, 25-37. 


\section{Apéndice}

Universidad Simón Bolívar

Departamento de Ciencia y Tecnología del Conocimiento

Departamento de Ciencias Económicas y Administrativas

Al responder este cuestionario, usted colabora con un proyecto de investigación sobre el consumo electrónico. Con las siguientes preguntas deseamos determinar la percepción que tiene usted acerca del uso de la tecnología para obtener bienes o servicios a través de internet.

Instrucciones:

Se recomienda responder con su primera impresión, sin pensar mucho la respuesta que se da e incluso si usted considera que es redundante; recuerde que no hay respuestas correctas o incorrectas. La información suministrada tendrá carácter anónimo y será tratada de manera confidencial.

Responda la opción escogida rellenando completamente el círculo y/o escribiendo en el espacio correspondiente. Recuerde indicar para cada opción si la operación es gratuita y/o comporta un pago.

1. Género: $\square$ Masculino $\square$ Femenino

2. Edad:

3. Profesión: Ocupación: Posgrado que cursa:

4. ¿En cuál nivel socio-económico se ubicaría usted?

\begin{tabular}{|c|c|c|c|c|c|c|c|c|c|c|c|c|c|c|c|c|c|c|}
\hline$\square$ & 1 & D & 2 & ב & 3 & 口 & 4 & $\square$ & 5 & D & 6 & $\square$ & 7 & D & 8 & D & 9 & 10 \\
\hline
\end{tabular}

5. Indique los sitios de internet que más utiliza:

$\begin{array}{llll}\text { Owww.terra.com } & \text { O www.amazon.com } & \text { Owww.altavista.com } & \text { Owww.google.com } \\ \text { Owww.aol.com } & \text { Owww.latinmail.com } & \text { Owww.hotmail.com } & \text { Owww.yahoo.com } \\ \text { Owww.tucine.com } & \text { O www.mercadolibre.com } & \text { Owww.porlapuerta.com } & \text { Owwwtucarro.com } \\ \text { Owww.ebay.com } & \text { O Otros } & \end{array}$

6. ¿Cuáles medios de pago dispone para compras por internet?

$\begin{array}{lll}\square \text { Depósito bancario } & \square \text { Tarjeta de crédito } & \square \text { E-Card } \\ \square \text { Transferencia bancaria } & \square \text { Otros } & \\ \end{array}$

7. iTiene tarjeta de crédito habilitada para compras por internet $\quad \square \mathrm{Si} \quad \square \mathrm{No}$

8. Persibe dificultades asociadas a la compra por internet?

En caso de respuesta, indique cuáles:

sigue

| Universitas Psychologica | V. 10 | No.1 | enero-abril | 2011 | 
9. Las siguientes afirmaciones están dirigidas a conocer su intención de realizar las operaciones electrónicas que se mencionan a continuación:

\begin{tabular}{|c|c|c|c|c|c|c|}
\hline En algunos de estos sitios: & Muy probable & Probable & Poco probable & Improbable & \multicolumn{2}{|c|}{$\begin{array}{c}\text { Gratis }(\mathrm{G}) \text { y/o } \\
\text { pago }(\mathrm{P})\end{array}$} \\
\hline Compraría los productos en oferta & घ & ב & ב & घ & GD & $\mathrm{P \square}$ \\
\hline Contrataría los servicios en oferta & D & $\square$ & $\square$ & 口 & G] & $\mathrm{P}$ \\
\hline Adquiriría la información en oferta & $\square$ & $\square$ & 口 & $\square$ & GD & $\mathrm{PD}$ \\
\hline Compraría productos piratas & $\square$ & $\square$ & $\square$ & $\square$ & GD & $\mathrm{Pa}$ \\
\hline Contrataría servicios clandestinos & $\square$ & $\square$ & 口 & $\square$ & GD & $\mathrm{PU}$ \\
\hline Adquiriría información esotérica & $\square$ & $\square$ & ב & $\square$ & GD & $\mathrm{P} \square$ \\
\hline Compraría productos de prestigio & ] & $\square$ & ] & 口 & GD & $\mathrm{PD}$ \\
\hline Contrataría servicios de calidad & $\square$ & $\square$ & $\square$ & $\square$ & GD & $\mathrm{PD}$ \\
\hline Adquiriría información relevante & $\square$ & $\square$ & $\square$ & $\square$ & GD & $\mathrm{Pa}$ \\
\hline Compraría productos de moda & $\square$ & $\square$ & $\square$ & $\square$ & GD & $\mathrm{Pa}$ \\
\hline Coptrataría servicios actualizados & $\square$ & D & $\square$ & $\square$ & GD & $\mathrm{Pa}$ \\
\hline Autorizaría mi tarjeta de crédito & $\square$ & $\square$ & $\square$ & $\square$ & G] & $\mathrm{P}$ \\
\hline
\end{tabular}

¿Qué otro tipo de operaciones tendría usted la intención de realizar a través de alguno de estos sitios?

Comentarios:

10. Las siguientes afirmaciones están dirigidas a conocer sus habilidades para realizar las operaciones electrónicas que se mencionan a continuación:

\begin{tabular}{|c|c|c|c|c|c|}
\hline En algunos de estos sitios puedo: & $\begin{array}{c}\text { Totalmente } \\
\text { en desacuerdo }\end{array}$ & $\begin{array}{l}\text { En desa- } \\
\text { cuerdo }\end{array}$ & \begin{tabular}{|c|}
$\begin{array}{c}\text { De } \\
\text { acuerdo }\end{array}$ \\
\end{tabular} & $\begin{array}{l}\text { Totalmente } \\
\text { de acuerdo }\end{array}$ & $\begin{array}{c}\text { Gratis }(G) \\
\text { y/o pago }(P)\end{array}$ \\
\hline Eliminaría las ofertas inesperadas & $\square$ & $\square$ & $\square$ & $\square$ & $\mathrm{GD} \quad \mathrm{P}$ \\
\hline Evitar la publicidad repentina & ] & $\square$ & ] & $\square$ & GD \\
\hline Elegir la información científica que necesito & $\square$ & $\square$ & 口 & $\square$ & GD \\
\hline Seleccionar ofertas que necesito & ] & 口 & $\square$ & $\square$ & $\mathrm{G} \square$ \\
\hline Elegir con quién chatear & 口 & $\square$ & 口 & 口 & $\mathrm{G} \square$ \\
\hline Inscribirme en cursos y membresías que necesito & $\square$ & $\square$ & $\square$ & $\square$ & $\mathrm{GD}$ \\
\hline Comprar los productos que busco & $\square$ & $\square$ & $\square$ & 口 & GD \\
\hline Contratar los servicios que me gustan & $\square$ & 口 & $\square$ & 口 & $\mathrm{GD}$ \\
\hline Encontrar ofertas especiales & $\square$ & $\square$ & ב & $\square$ & GD \\
\hline Bajar imágenes, sonidos y videos & $\square$ & $\square$ & $\square$ & $\square$ & GD \\
\hline
\end{tabular}

¿Qué otro tipo de operaciones cree usted saber hacer en alguno de estos sitios?

Comentarios:

sigue 
11. Las siguientes afirmaciones están dirigidas a conocer su apreciación sobre la utilidad de usar internet para realizar las operaciones que se mencionan a continuación:

\begin{tabular}{|c|c|c|c|c|c|c|}
\hline En algunos de estos sitios: & Muy útil & Útil & Poco útil & Inútil & \multicolumn{2}{|c|}{$\begin{array}{c}\text { Gratis }(\mathrm{G}) \text { y/o } \\
\text { pago }(\mathrm{P})\end{array}$} \\
\hline Se compran las cosas que uno busca & 口 & $\square$ & $\square$ & $\square$ & GD & $\mathrm{Pa}$ \\
\hline Se chatea con tipo de persona que uno busque & 口 & $\square$ & $\square$ & $\square$ & G] & $\mathrm{P} \square$ \\
\hline Se consulta información académica de interés & $\square$ & $\square$ & $\square$ & $\square$ & $\mathrm{GD}$ & P \\
\hline Se contrata los servicios que uno busque & 口 & 口 & 口 & 口 & G] & $\mathrm{Pa}$ \\
\hline Se mejoran los criterios de compra & 口 & $\square$ & 口 & 口 & $\mathrm{GD}$ & $\mathrm{PD}$ \\
\hline Se desarrollan habilidades comunicativas & $\square$ & $\square$ & 口 & $\square$ & G] & P \\
\hline Se aprende a encontrar cualquier tipo de información & 口 & 口 & ב & 口 & GD & $\mathrm{PD}$ \\
\hline Se compra fácilmente & $\square$ & ] & $\square$ & ] & G] & $\mathrm{PD}$ \\
\hline Se contratan servicios fácilmente & 口 & $\square$ & 口 & 曰 & $\mathrm{GD}$ & $\mathrm{PD}$ \\
\hline Se hacen fácilmente inscripciones a cursos/membresías & a & 口 & a & ב & Ga & P \\
\hline
\end{tabular}

Por cuáles otras razones cree usted que es útil usar alguno de estos sitios?

Comentarios:

12. Lea cada una de las afirmaciones y seleccione la respuesta que refleje mejor la frecuencia con la que usted ha realizado las operaciones que se mencionan a continuación, durante los últimos seis meses:

\begin{tabular}{|c|c|c|c|c|c|}
\hline \multirow{2}{*}{ En algunos de estos sitios: } & \multicolumn{4}{|c|}{ Veces mensuales } & \multirow{2}{*}{$\begin{array}{l}\text { Gratis }(G) \\
\text { y/o pago }(P)\end{array}$} \\
\hline & De 1 a 9 & De 10 a 19 & De 20 a 29 & 30 o más & \\
\hline He comprado videos, imágenes, películas & $\square$ & $\square$ & $\square$ & $\square$ & $\mathrm{G} \square \quad \mathrm{P} \square$ \\
\hline He contratado servicios & 口 & 口 & $\square$ & $\square$ & Ga \\
\hline He consultado información académica & 口 & 口 & $\square$ & $\square$ & GD \\
\hline He comprado melodías, ringtones o música & $\square$ & $\square$ & $\square$ & $\square$ & G] \\
\hline He contratado servicios de telefonía & 口 & 口 & 口 & $\square$ & GD \\
\hline En estos sotios he comprado libros & $\square$ & $\square$ & $\square$ & $\square$ & GD \\
\hline $\begin{array}{l}\text { En estos sotios he adquirido boletos para espec- } \\
\text { táculos }\end{array}$ & 口 & 口 & $\square$ & $\square$ & $\mathrm{G} \square$ \\
\hline En estos sitios he adquirido hardware.software & $\square$ & $\square$ & $\square$ & $\square$ & $\mathrm{G} \square$ \\
\hline He comprado boletos para viajar & $\square$ & $\square$ & $\square$ & $\square$ & GD \\
\hline He contratado servicios bancarios & 口 & 口 & 口 & 口 & GD \\
\hline Me he inscrio en cursos/membresías & 口 & 口 & $\square$ & $\square$ & GD \\
\hline
\end{tabular}

¿Qué otro tipo de operaciones ha realizado a través de alguno de estos sitios?

Comentarios: 
